\title{
ANÁLISE DE PERFIS DE AÇO DE SEÇÃO ABERTA SUBMETIDOS À ESFORÇOS DE TORÇÃO
}

\author{
MANICA, GUSTAVO \\ Laboratorista \\ Universidade do Vale do Rio dos Sinos \\ Rio Grande do Sul; Brasil \\ gustavo.manica@hotmail.com \\ LEITE, RODRIGO \\ Engenheiro Civil \\ Universidade do Vale do Rio dos Sinos \\ Rio Grande do Sul; Brasil \\ rodrigosarmentoleite@gmail.com
}

\author{
BOLINA, FABRÍCIO \\ Professor e Engenheiro Civil \\ Universidade do Vale do Rio dos Sinos \\ Rio Grande do Sul; Brasil \\ fabriciolb@unisinos.br
}

\section{RESUMO}

Nas estruturas de aço, a ligação estrutural entre vigas secundárias e vigas principais é de significativa importância no tocante à estabilidade e segurança de uma edificação. Por se tratarem de locais de transferências de cargas e esforços, as ligações entre estes elementos, dependendo do modelo de cálculo admitido, podem ser pontos de momentos torsores concentrados, as vezes de grandes magnitudes, podendo promover tensões indesejadas oriundos do empenamento do perfil. Do exposto, o presente trabalho visa realizar uma revisão da literatura internacional que expressa os critérios de dimensionamento e tratamento dos efeitos de momento torsor, através do método das equações diferenciais, método simplificado e com o auxílio de uma ferramenta computacional uma análise por elementos finitos. Espera-se que, com este trabalho, seja determinado a validade do método simplificado para utilização prática e rotineira no desenvolvimento de projetos de estruturas, propondo, assim, sugestões de rotinas de cálculo aos projetistas, haja visto a lacuna normativa sobre o assunto.

Palavras-chave: Estruturas de aço, Torção, Empenamento, Vigas de Seção Aberta.

\begin{abstract}
In steel structures, the structural connection between secondary and main beams is of significant importance regarding the stability and safety of a building. Because these are places of load and efforts transfer, the connections between these elements, depending on the calculation model, can be points of concentrated torsional moments, sometimes of great magnitudes, and may promote undesired tensions derived from profile warping. Thus, the present study aims to perform a review of the international literature that expresses the criteria for sizing and treatment of torsional moment effects, using the differential equation method, simplified method and, with the aid of a computational tool, a finite element analysis. It is hoped that, with this study, the validity of the simplified method for practical and routine use in the development of structural projects will be determined, thus proposing calculation suggestions to designers, having seen the normative gap on the issue.
\end{abstract}

Keywords: Steel Structures, Torsion, Warping, Open Section Beams.

\section{INTRODUÇÃO}

O dimensionamento e/ou verificação à torção de perfis laminados é feito em condições específicas de um projeto estrutural. Por exemplo, na utilização de elementos de fachada pré-fabricados fixados excentricamente em uma viga, ou a ligação engastada de uma viga secundária em uma viga principal (HUGHES; ILES; MALIK, 2011). Isto reflete na inexistência de critérios de cálculo a este tipo de solicitação em vigas de seção aberta em normativas internacionais, como a AS 4100 (AS, 2012), NBR 8800 (ABNT, 2008), AISC 360-16 (AISC, 2016), IS-800 (BIS, 2007) e EN1993 (EN, 2005). 
É sabido que na torção obtem-se um aproveitamento maior de material ao utilizarmos uma seção vazada, pois as tensões máximas ocorrem sempre nas fibras mais distantes do centro de rotação da peça (HIBBELER, 2007). Do exposto, quando uma peça que não possua uma seção transversal circular e/ou fechada está sujeito à torção, esta irá sofrer empenamento, gerando deformações laterais e tensões normais e cisalhantes adicionais na mesa. No caso específico de um perfil I, o empenamento fará com que as mesas se comportem de maneira similar à uma viga bi-apoiada, ou seja, gera-se tensões adicionais normais e de cisalhamento na seção da peça (ESTABROOKS; GRONDIN, 2008).

Durante a fase de concepção de um projeto de estrutura metálica, ao tentar adequar-se às limitações arquitetônicas de um empreendimento, o engenheiro pode deparar-se com uma situação que provoque solicitações de torção em uma viga. Devido à complexidade de uma análise refinada de torção para os perfis mais utilizados, existe uma tendência de o projetista, ao se encontrarem nesta situação, reavaliar e modificar a sua concepção inicial de modo a evitar este tipo de solicitação. Inclusive, existem normas de projetos de estruturas de aço com sugestões para evitar situações comuns de torção em vigas (SEABURG; CARTER, 2003).

Em estruturas convencionais, o momento torsor atuante sobre uma viga é de baixa magnitude, podendo ser desprezado no cálculo. Entretanto, em ocasiões nas quais este esforço é significativo, torna-se necessário realizar uma análise refinada para verificar a segurança estrutural do mesmo (HOTCHKISS, 1966). Todavia, nota-se uma limitação de normas de projeto que possuem esta abordagem relacionada ao momento torsor, fato que é justificado pela complexidade do cálculo e a frequência em que ocorre situações que necessitam esta análise (TAHAIR; PI, 1997).

Portanto, para estabelecer critérios de cálculo, o projetista pode buscar referências no Design Guide Series 9 (SEABURG; CARTER, 2003) e no Design of Steel Beams in Torsion (LIN, 1997), guias de dimensionamento de vigas sujeitas à torção reconhecidos pela AISC 360-16 (AISC, 2016) e EN1993 (EN, 2005), respectivamente. Ambos os guias de dimensionamento abordam o método das equações diferenciais em regime elástico, este método torna-se complexo e demorado a ser utilizado manualmente.

Em virtude da complexidade do método das equações diferenciais, desenvolveu-se um método simplificado, onde se transforma o momento torsor atuante em um binário de forças coplanares e a viga passa a ser admitida como sendo sujeita à flexão. Porém, devido à esta simplificação, este método não apresenta resultados aproximados à uma situação real, sendo estes demasiadamente majorados ou minorados em função do modelo estrutural. Com isto, para que este método possa ter maior aproximação à valores reais, é necessário a utilização de um fator de correção $\beta$.

Do exposto, o presente trabalho visa realizar o dimensionamento e verificação estrutural de uma viga de aço com perfil I sujeita a um momento torsor concentrado, simulando aquele proveniente da ligação engastada de uma viga secundária, através do método das equações diferenciais, de acordo com ambos os guias e através do método simplificado. Pretende-se validar como mecanismo de verificação manual o uso do método simplificado, coibindo na menor complexibilidade do cálculo de torsão.

\section{METODOLOGIA}

O modelo de cálculo considerado será de uma viga principal com um vão de 8 metros. No centro do vão se tem uma viga secundária com um vão de 6 metros, que recebe carga da laje do pavimento, transferindo seu momento fletor para a viga principal através de um engaste idealizado perfeitamente rígido, ou seja, a totalidade do momento fletor será transferido como momento torsor.

A laje do pavimento foi considerada composta de concreto com uma espessura de $15 \mathrm{~cm}$, carga de uso de $1,50 \mathrm{kN} / \mathrm{m}^{2} \mathrm{e}$ vão contribuinte para a viga secundária de 3 metros. Assim, a viga secundária será sujeita a uma carga uniformemente distribuída de $5,25 \mathrm{kN} / \mathrm{m}$, desprezando-se o peso próprio da viga. O momento torsor atuante na viga principal é de 15,75 $\mathrm{kNm}$.

Para o dimensionamento foi utilizado o método das equações diferenciais, reconhecidos por normativas internacionais, e pelo método simplificado utilizando o fator de correção $\beta$. 


\subsection{Método das equações diferenciais}

Arbitrou-se o perfil laminado de bitola comercial W360x51 em aço ASTM 572 Gr.50 para realizar o dimensionamento. Com isso, utilizou-se as propriedades geométricas e mecânicas apresentados na Tabela 1.

Tabela 1 - Propriedades do perfil W360x51

\begin{tabular}{c|c|c|c}
\hline \multicolumn{2}{c|}{ Propriedades geométricas } & \multicolumn{2}{c}{ Propriedades geométricas } \\
\hline Altura total da seção transversal $(\mathrm{mm})$ & 355 & Raio de giração - y $(\mathrm{cm})$ & 3.87 \\
\hline Largura da mesa $(\mathrm{mm})$ & 171 & Módulo de resistência plástico $-\mathrm{y}\left(\mathrm{cm}^{3}\right)$ & 174.7 \\
\hline Espessura da alma $(\mathrm{mm})$ & 7.2 & Raio de giração - $\mathrm{t}(\mathrm{cm})$ & 4.49 \\
\hline Espessura da mesa $(\mathrm{mm})$ & 11.6 & Momento polar de inércia $\left(\mathrm{cm}^{4}\right)$ & 24.65 \\
\hline Distância entre as faces das mesas $(\mathrm{mm})$ & 332 & Constante de empenamento $\left(\mathrm{cm}^{6}\right)$ & 284994 \\
\hline Altura total da alma $(\mathrm{mm})$ & 308 & Fator de empenamento na mesa $\left(\mathrm{cm}^{2}\right)$ & 147 \\
\hline Área total da seção transversal $\left(\mathrm{cm}^{2}\right)$ & 64.8 & Momento estático - empenamento $\left(\mathrm{cm}^{4}\right)$ & 726 \\
\hline Momento de inércia $-\mathrm{x}\left(\mathrm{cm}^{4}\right)$ & 14222 & \multicolumn{2}{c}{ Propriedades mecânicas } \\
\hline Módulo de resistência elástico $-\mathrm{x}\left(\mathrm{cm}^{3}\right)$ & 801.2 & Módulo de elasticidade $(\mathrm{MPa})$ & 210000 \\
\hline Raio de giração $-\mathrm{x}(\mathrm{cm})$ & 14.81 & Módulo de elasticidade transv. $(\mathrm{MPa})$ & 80769.23 \\
\hline Módulo de resistência plástico $-\mathrm{x}\left(\mathrm{cm}^{3}\right)$ & 899.5 & Resistência ao escoamento - aço $(\mathrm{MPa})$ & 345 \\
\hline Momento de inércia $-\mathrm{y}\left(\mathrm{cm}^{4}\right)$ & 968 & Resistência à tração - aço $(\mathrm{MPa})$ & 450 \\
\hline Módulo de resistência elástico $-\mathrm{y}\left(\mathrm{cm}^{3}\right)$ & 113.3 & Resistência ao escoamento - aço $(\mathrm{MPa})$ & 207 \\
\hline
\end{tabular}

A partir destes calculou-se o coeficiente de flexão torcional e a relação deste com o vão da viga, apresentados, respectivamente, na Equação 1 e na Equação 2. Sendo E: módulo de elasticidade, $\mathrm{C}_{\mathrm{w}}$ : constante de empenamento, G: módulo de elasticidade transversal, J: momento polar de inércia, a: constante de flexão torcional e L: vão da viga.

$$
\begin{gathered}
a=\sqrt{\frac{E * C_{w}}{G * J}}: a=\sqrt{\frac{210000 * 284994}{80769.23 * 24.65}} \therefore a \cong 173,38 \mathrm{~cm} \\
\frac{L}{a}=\frac{800}{173,38} \div \frac{L}{a} \cong 4,61
\end{gathered}
$$

De posse do modelo estrutural determinou-se que o dimensionamento deverá ser realizado utilizando o ábaco do Caso 03 de torque concentrado em membros com extremidades rotuladas. Ambos os guias de dimensionamentos reconhecidos por normativas internacionais, AISC 360-16 (AISC, 2016) e EN1993 (EN, 2005), apresentam soluções para este modelo estrutural.

Os ábacos fornecidos na Design Guide Series 9 (SEABURG; CARTER, 2003) se adequam ao modelo estrutural especificado são denominados como o Caso 3. Estes possuem no seu eixo horizontal z/L, onde deve-se localizar a seção do vão da viga desejada, e no eixo vertical a solução da função torcional para $\phi$ (ângulo de rotação da seção transversal), $\phi$ ' (primeira derivada do ângulo de rotação), $\phi$ ' (segunda derivada do ângulo de rotação) e $\phi$ "' (terceira derivada do ângulo de rotação), sendo estes separados em ábacos individuais, estão plotadas as curvas referentes às soluções para diferentes relações L/a.

Estes ábacos estão apresentados, na Figura 1a, Figura 1b, Figura 1c e Figura 1d, respectivamente, e as funções com suas devidas soluções apresentam-se na Equação 3, Equação 4, Equação 5 e Equação 6, respectivamente. Sendo T: momento de torção. 


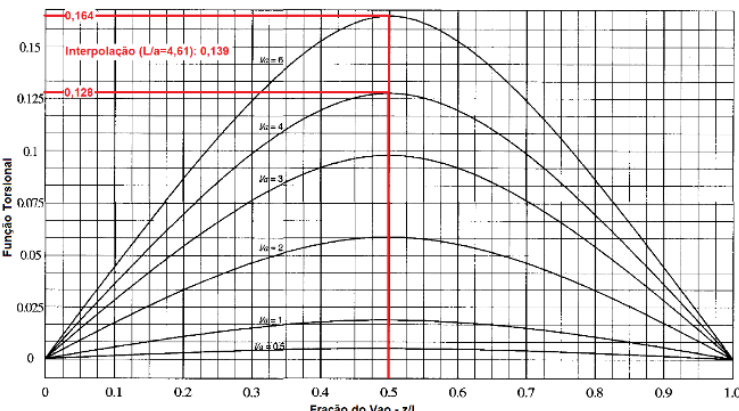

(a)

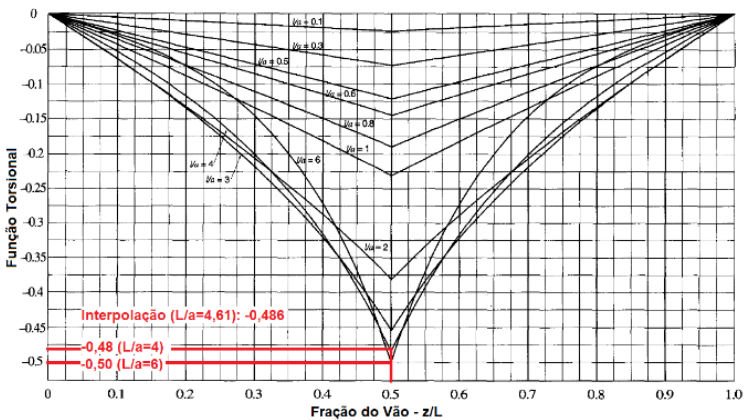

(c)

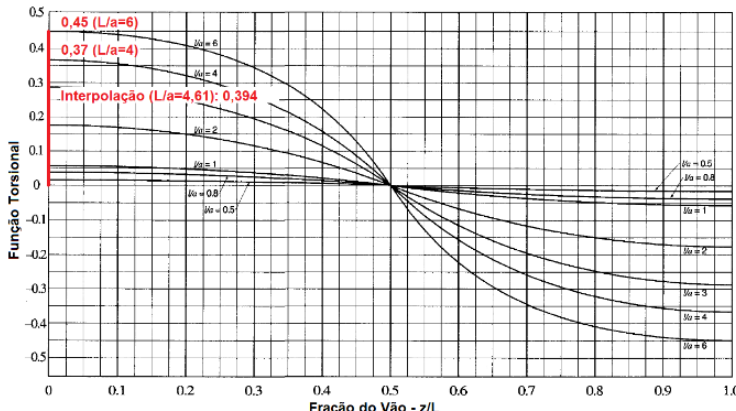

(b)

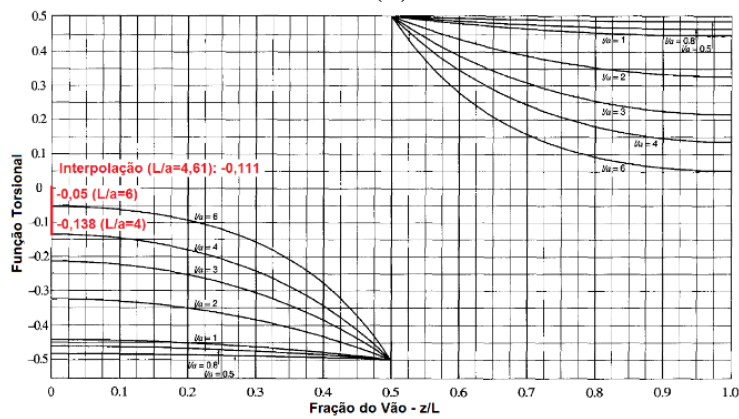

(d)

Figura 1: Ábaco para (a) $\phi$ Caso 3, (b) $\phi^{\prime}$ Caso 3, (c) $\phi^{\prime \prime}$ Caso 3 e (d) $\phi '$, ' Caso 3

$$
\begin{gathered}
\phi *\left(\frac{G * J}{T} * \frac{1}{L}\right)=0,139 \div \phi=\frac{0,139}{\left(\frac{8076,923 * 24,65}{1575 * 800}\right)} \div \phi \cong 0,880 \\
\phi^{\prime} *\left(\frac{G * J}{T}\right)=0,394 \div \phi^{\prime}=\frac{0,394}{\left(\frac{8076,923 * 24,65}{1575}\right)} * \phi^{\prime} \cong 0,00312 \mathrm{~cm} \\
\phi^{\prime \prime} *\left(\frac{G * J}{T} * \frac{L}{a}\right)=-0,486 \div \phi^{\prime \prime}=\frac{-0,486}{\left(\frac{8076,923 * 24,65 * 4,61}{1575}\right)} * \phi^{\prime \prime}=-0,000834 \mathrm{~cm}^{-2} \\
\phi^{\prime \prime \prime} *\left(\frac{G * J}{T} *\left(\frac{L}{a}\right)^{2}\right)=-0,111 * \phi^{\prime \prime \prime}=\frac{-0,111}{\left(\frac{8076,923 * 24,65}{1575} * 4,61^{2}\right)} * \phi^{\prime \prime \prime} \cong-0,0000413 \mathrm{~cm}^{-3}
\end{gathered}
$$

Nota-se que no centro do vão, independentemente da relação L/a, deve-se considerar o valor da função torcional como sendo $-0,5$, com isso, determinou-se que $\phi$ "' com o valor de $-0,000186$.

De posse destes valores, pode-se calcular as tensões atuantes na seção transversal da viga. O cálculo dos valores máximos de $\tau_{\mathrm{t}}$ (tensão de cisalhamento de torção de Saint Venant), $\tau_{\mathrm{w}}$ (tensão de cisalhamento de torção de empenamento) e $\sigma_{\mathrm{w}}$ (tensão normal de torção de empenamento) apresenta-se na Equação 7, Equação 8 e Equação 9 , respectivamente.

$$
\begin{gathered}
\tau_{t}=8076,923 * 0,72 * 0,00312: \tau_{t}=18,14 \frac{\mathrm{kN}}{\mathrm{cm}^{2}} \\
\tau_{w}=\frac{-21000 * 726}{1,16} *-0,000186: \tau_{w}=2444,62 \frac{\mathrm{kN}}{\mathrm{cm}^{2}} \\
\sigma_{w}=21000 * 147 *-0,000834 \therefore \sigma_{w}=-2574,56 \frac{\mathrm{kN}}{\mathrm{cm}^{2}}
\end{gathered}
$$

\subsection{Método simplificado}

Para a determinação das tensões normais atuantes na seção através do método simplificado, utilizou-se os valores de $\beta$ (coeficiente de correção) desenvolvidas e tabeladas por Lin (1997). Primeiramente, considerando a relação L/a já 
determinada no método das equações diferenciais, é necessário simplificar o momento torsor atuante pelo binário de forças aplicados perpendicularmente às mesas do perfil e assim, calcularmos o momento fletor simplificado solicitante $\left(\mathrm{M}_{\mathrm{o}}\right)$. Apresenta-se este desenvolvimento na Equação 10.

$$
M_{o}=\frac{\left(\frac{1575}{35,5}\right) * 800}{4} \div M_{o} \cong 88,73 \mathrm{kNm}
$$

Do exposto, para a utilização das tabelas do fator $\beta$, é necessário, de modo similar aos ábacos do método das equações diferenciais, ter como dados de entrada a relação L/a, a fração do vão onde está aplicada o momento torsor e a fração do vão onde se deseja analisar. No caso de uma relação L/a não coincidir com aqueles fornecidos na tabela, é permitido a realização de interpolação linear com os valores imediatamente superior e inferior. Apresenta-se na Tabela 2 os valores do fator $\beta$, isolados os valores necessários para o presente trabalho, para um modelo estrutural de viga bi-rotulada com um momento torsor concentrado.

Tabela 2 - Valores isolados para o fator $\beta$

\begin{tabular}{c|c|c}
\hline $\mathbf{L} / \mathbf{a}$ & $\boldsymbol{\alpha}$ & $\boldsymbol{\beta}$ \\
\hline 4 & 0.5 & 0.48201 \\
\hline 4.61 & 0.5 & 0.428356 \\
\hline 5 & 0.5 & 0.39465 \\
\hline
\end{tabular}

Calculado o fator $\beta$, podemos determinar a magnitude do momento fletor corrigido solicitante nas mesas do perfil e, posteriormente as tensões normais atuantes. Apresenta-se na Equação 11 e Equação 12 as soluções para o momento $\mathrm{M}_{\mathrm{T}}$ (momento fletor corrigido na mesa) e a tensão $\sigma_{\mathrm{w}}$ (tensão normal de torção de empenamento), respectivamente.

$$
\begin{gathered}
M_{T}=0,428356 * 88,73 \therefore M_{T}=38,01 \mathrm{kNm} \\
\sigma_{w}=\frac{2 * 3801}{113,3} \therefore \sigma_{w}=67,10 \frac{\mathrm{kN}}{\mathrm{cm}^{2}}
\end{gathered}
$$

\subsection{Modelagem computacional}

Foi realizado um ensaio computacional com a utilização de um software de análise estrutural pelo método dos elementos finitos Autodesk Robot Structural Analysis Professional 2018. Assim, tornou-se possível observar a distribuição de tensões ao longo da viga através do mapeamento destas em torno do modelo tridimensional.

O perfil metálico W360x51 foi modelado criando um painel de aço com as devidas dimensões da seção transversal e feito a extrusão do mesmo para o comprimento total da viga de 8 metros, gerando um sólido com as propriedades mecânicas definidas de acordo com o aço ASTM 572 Gr.50. Nas extremidades, foi definido como condição de contorno apoios com translação impedida nos 3 eixos globais e rotação impedida somente nos eixos globais X e Z, sendo a rotação em torno do eixo Y livre, de modo à similar a condição de rotulado para torção.

Através da ferramenta de definição de malhas do software, foi utilizado uma malha de 1 milímetro por 1 milímetro e, posteriormente aplicado um momento torsor nodal concentrado de $15,75 \mathrm{kNm}$ no nó central do modelo. Assim, simulou-se computacionalmente o modelo estrutural definido.

Gerado o modelo, foi feito o processamento do software e obtidos os mapas para as tensões normal e cisalhante solicitantes no perfil. Para as tensões normais, foram consideradas somente aquelas atuando no eixo Y global, ou seja, aquelas que estão solicitando perpendicularmente à seção transversal da peça e não longitudinal. As tensões cisalhantes oriundos do empenamento foram analisadas no eixo Y global, e as oriundos da torção de Saint Venant no eixo X global. Apresenta-se na Figura 2a, Figura $2 \mathrm{~b}$ e Figura $2 \mathrm{c}$ os mapas das $\sigma_{\mathrm{w}}$ (tensão normal de torção de empenamento), $\tau_{\mathrm{w}}$ (tensão de cisalhamento de torção de empenamento) e $\tau_{\mathrm{t}}$ (tensão de cisalhamento de torção de Saint Venant) com as suas escalas de magnitudes, respectivamente. 


\section{CBPAT 2020 ANAIS

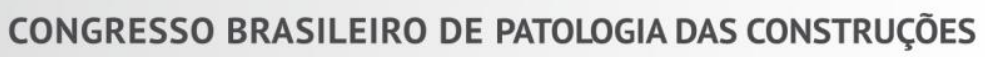 \\ DE 15 A 17 DE ABRIL | FORTALEZA - CE

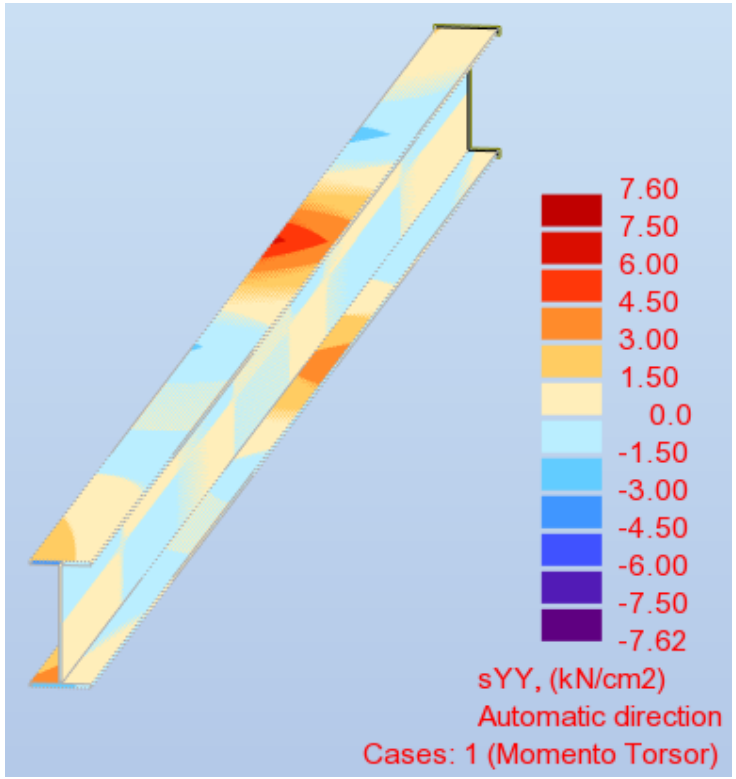

(a)

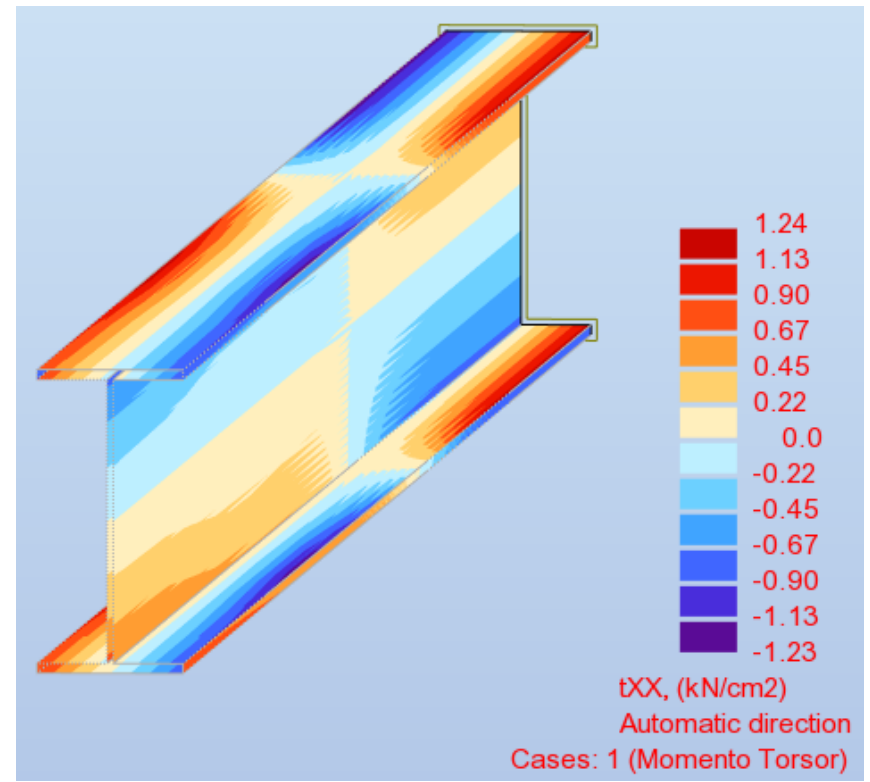

(b)

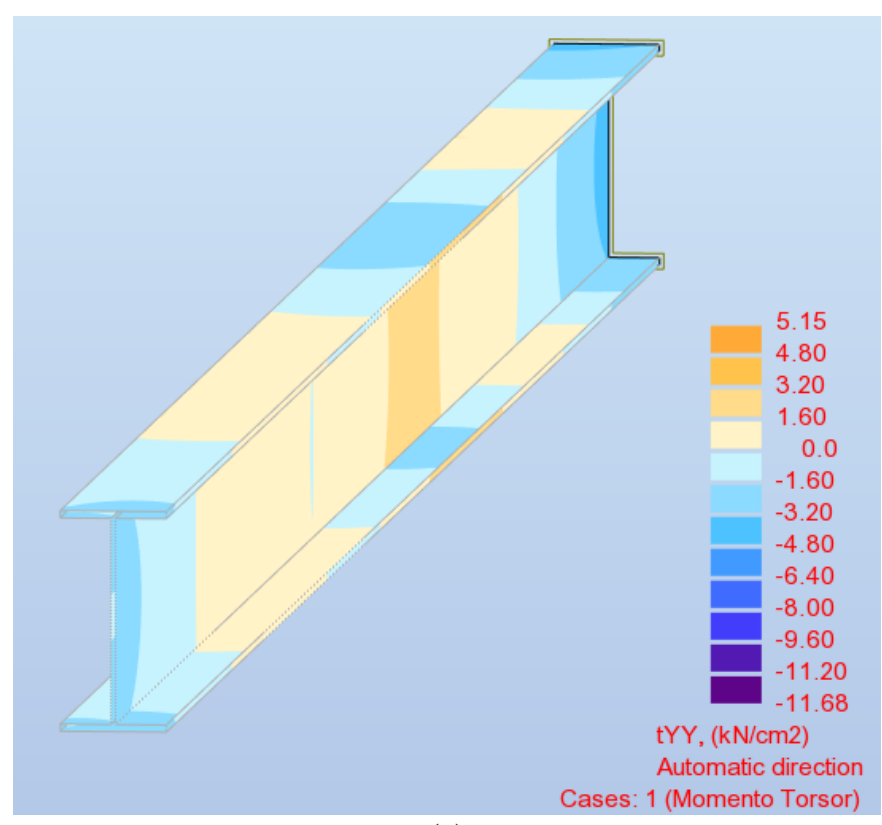

(c)

Figura 2: Mapa das tensões (a) normais $\sigma_{\mathrm{w}}$, (b) cisalhamento $\tau_{\mathrm{w}} \mathrm{e}$ (c) cisalhamento $\tau_{\mathrm{t}}$

\section{APRESENTAÇÃO E DISCUSSÃO DOS RESULTADOS}

$\mathrm{Na}$ Tabela 3, estão apresentados os valores das tensões solicitantes calculados através do método das equações diferenciais, do método simplificado e da modelagem computacional. 
Tabela 3 - Comparativo das tensões solicitantes

\begin{tabular}{|c|c|c|c|c|}
\hline \multirow[b]{2}{*}{ Posição na viga } & \multirow{2}{*}{ 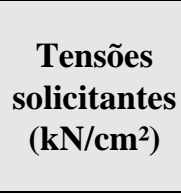 } & \multicolumn{3}{|c|}{ Método de cálculo } \\
\hline & & $\begin{array}{c}\text { Método das } \\
\text { equações } \\
\text { diferenciais }\end{array}$ & $\begin{array}{c}\text { Método } \\
\text { simplificado }\end{array}$ & $\begin{array}{c}\text { Modelagem } \\
\text { computacional }\end{array}$ \\
\hline \multirow{3}{*}{ Centro do vão } & $\sigma_{\mathrm{w}}$ & 2574,56 & 67,10 & 7,62 \\
\hline & $\tau_{\mathrm{w}}$ & 2444,62 & Não determinado & 0 \\
\hline & $\tau_{\mathrm{t}}$ & 0 & Não determinado & 11,68 \\
\hline \multirow{3}{*}{ Sobre apoios } & $\sigma_{\mathrm{w}}$ & 0 & 0 & 0 \\
\hline & $\tau_{\mathrm{w}}$ & 542,81 & Não determinado & 1,24 \\
\hline & $\tau_{t}$ & 18,14 & Não determinado & 1,60 \\
\hline
\end{tabular}

No método das equações diferenciais foi analisado somente o centro do vão e as extremidades, pois nestes locais obtemos as maiores tensões normais e de cisalhamento, respectivamente. Pode-se observar que, como esperado, as tensões normais oriundos do empenamento das mesas são os de magnitudes mais elevadas, e consequentemente, os mais significativos.

O método simplificado não contempla a determinação das tensões tangenciais atuantes tanto devido à torção de Saint Venant ou ao empenamento. Isto se deve à predominância da tensão normal sobre as demais e por este método ter utilização rápida em situações práticas.

Os resultados obtidos através da modelagem computacional apresentaram as menores magnitudes entre os métodos analisados. Nota-se no mapa de tensões normais que, como nos outros métodos, as maiores tensões normais ocorrem no ponto de aplicação do momento torsor e que estes tendem a reduzir ao aproximarem-se dos pontos de apoio onde os mesmos apresentam valores nulos. No caso das tensões de cisalhamento, observa-se que ocorre o efeito contrário. As tensões máximas se encontram sobre os apoios e os nulos no ponto de aplicação. Esta distribuição de tensão confirma a tendência da mesa, quando torcida, a se comportar de modo muito similar à uma viga que recebe cargas verticais.

Podemos observar que para o modelo estrutural adotado, houve uma divergência de grande magnitude no que diz respeito às tensões normais solicitantes entre todos os métodos analisados. O método das equações diferenciais apresenta um valor aproximadamente 38 vezes mais expressivo que aquele obtido pelo método simplificado e aproximadamente 338 vezes mais que a análise computacional. Nota-se a tendência que a tensão de maior magnitude em todos os métodos é a tensão normal gerada pelo empenamento das mesas.

Devido ao método simplificado não abordar a determinação das solicitações tangenciais que atuam na seção, serão considerados somente aqueles obtidos pelo método das equações diferenciais e a análise computacional. Pode-se observar que ocorreu um comportamento inverso no que diz respeito à distribuição destas tensões nestes dois modelos, as maiores tensões se encontram na análise computacional. Nota-se, também, que no modelo computacional as tensões de cisalhamento mais significativos são aquelas provenientes da torção de Saint Venant, enquanto no método das equações diferenciais, esta solicitação gera tensões insignificantes em relação às demais.

\section{CONCLUSÃO}

A análise numérica do modelo estrutural proposto através do método das equações diferenciais e o método simplificado permitiu um estudo satisfatório no que diz respeito à teoria de vigas de aço com perfil I sujeitos à torção. Principalmente no que tange o fenômeno do empenamento das mesas e na redução da resistência torcional devido a isto. $\mathrm{O}$ modelo computacional mostrou-se satisfatório na demonstração dos mapas de tensões, possibilitando a boa visualização na distribuição dos esforços ao longo do comprimento e seção transversal da viga.

No que diz respeito aos resultados das análises, estes não demonstraram convergência de magnitudes nas tensões. Contra as expectativas, de acordo com a revisão bibliográfica, o método das equações diferenciais apresentou valores 
demasiadamente mais significativos que os demais métodos. No entanto, houve concordância unânime, confirmando a literatura, em todos os métodos de que a tensão com valores mais expressivos, relativamente com as demais obtidos pelo mesmo método, é a tensão normal nas mesas devido ao empenamento da mesa. Justamente por esta tendência de a peça sofrer empenamento que haja a pouca utilização desta geometria de perfil para situações onde ocorrerá torção.

Ao analisar-se as tensões de cisalhamento devido à torção de Saint Venant, observou-se a divergência no comportamento destas tensões entre os métodos que contemplam esta análise. De acordo com o mapa das tensões através do modelo computacional, o cisalhamento tem seus valores máximos no centro do vão, ou seja, no ponto de aplicação da carga. No entanto, os resultados obtidos pelo método das equações diferenciais demonstram o comportamento contrário, ocorrendo as tensões máximas sobre os apoios.

Por fim, o estudo realizado contribuiu para a melhor compreensão e entendimento da distribuição dos esforços ao longo de um elemento metálico com seção aberta quando o mesmo se encontra sujeito à torção. Porém, o presente trabalho teve como objetivo a validação do modelo simplificado para uma aplicação prática no uso de um engenheiro civil e, no entanto, para as condições de contorno e modelo estrutural aqui analisados, não obteve-se resultados adequados para determinar esta confirmação.

\section{REFERÊNCIAS}

HUGHES, A. F.; ILES D. C.; MALIK A. S. Design of steel beams in torsion. Askot: SCI, 2011.

STANDARDS AUSTRALIA INTERNATIONAL. AS 4100-1998/Amdt 1-2012: Steel structures. SAI, 2012.

ASSOCIAÇÃO BRASILEIRA DE NORMAS TÉCNICAS. NBR 8800: Projeto de estruturas de aço e de estruturas mistas de aço e concreto de edifícios. Rio de Janeiro, 2008.

AMERICAN INSTITUTE OF STEEL CONSTRUCTION. AISC 360-16: Specification for Structural Steel Buildings. Chicago, 2016.

BUREAU OF INDIAN STANDARDS. IS 800: General construction in steel - Code of practice. New Delhi, 2007.

EUROPEAN STANDARDS. EN1993 1-1: Eurocode 3: Design of Steel Structures - Part1-1: General Rules and Rules for buildings. Bruxelas, 2005.

HIBBELER R. C. Resistência dos materiais. 7. ed. São Paulo: Pearson Education do Brasil, 2010.

ESTABROOKS B. G.; GRONDIN G. Y. Combined Bending and Torsion of Steel I-Shaped Beams. University of Alberta. Edmonton, 2008.

SEABURG P. A.; CARTER C. J. AISC Steel Design Guide Series 9, Torsional Analysis of Structural Steel Members. Chicago: AISC, 2003.

HOTCHKISS J. G. Torsion of Rolled Steel Sections in Building Structures. AISC Engineering Journal, Chicago, v. 3, p. 19-45, 1966.

TAHAIR N. S.; PI Y. L. Torsion, bending and buckling of steel beams. Engineering Structures, Sydney, v. 19, p. 372-377, 1997.

LIN P. H. Simplified Design for Torsional Loading of Rolled Steel Members. AISC Engineering Journal, Chicago, p. $98-107,1977$. 